\title{
Controlling the dynamic range of a Josephson parametric amplifier
}

\author{
Christopher Eichler ${ }^{*}$ and Andreas Wallraff
}

\author{
${ }^{*}$ Correspondence: \\ eichlerc@phys.ethz.ch \\ Department of Physics, ETH Zürich, \\ Zürich, 8093, Switzerland
}

\begin{abstract}
One of the central challenges in the development of parametric amplifiers is the control of the dynamic range relative to its gain and bandwidth, which typically limits quantum limited amplification to signals which contain only a few photons per inverse bandwidth. Here, we discuss the control of the dynamic range of Josephson parametric amplifiers by using Josephson junction arrays. We discuss gain, bandwidth, noise, and dynamic range properties of both a transmission line and a lumped element based parametric amplifier. Based on these investigations we derive useful design criteria, which may find broad application in the development of practical parametric amplifiers.
\end{abstract}

\section{Introduction}

Due to the rapidly evolving field of quantum optics and information processing with superconducting circuits the interest in low-noise amplifiers has dramatically increased in the past five years and has lead to a body of dedicated research on Josephson junction based amplifiers [1-15]. The most successful quantum limited detectors which have so far been realized in the microwave frequency range are based on the principle of parametric amplification [16-19]. Josephson parametric amplifiers (JPAs) have not only been used to generate squeezed radiation $[6,13,20-22]$, but moreover enabled the realization of quantum feedback and post-selection based experiments [23-26], the efficient displacement measurement of nanomechanical oscillators [27] and the exploration of higher order photon field correlations $[28,29]$.

While JPAs have been demonstrated to operate close to the quantum limit, their performance is to date mostly limited by their relatively small dynamic range, i.e. the saturation of the gain for large input signals. Here, we discuss the control of the dynamic range by making use of Josephson junctions arrays in the parametric amplifier circuit, which we have already employed in recent experiments $[29,30]$. After reviewing the principles of parametric amplification we discuss bandwidth and noise constraints in dependence on the circuit design, based on which we derive simple strategies for optimized circuit design.

\section{Principles of parametric amplification}

\subsection{Parametric processes at microwave frequencies}

In quantum optics the word parametric is used for processes in which a nonlinear refractive medium is employed for mixing different frequency components of light. Such processes are parametric in the sense that a coherent pump field, applied to a nonlinear 
medium, modulates its refractive index, which appears as a parameter in a semi-classical treatement. This time-varying parameter is affecting modes with frequencies detuned from the frequency of the pump field and can stimulate their population with photons. The energy for creating these photons is provided by the pump field.

The refractive index in optics is equivalent to the impedance of electrical circuits. In order to realize parametric processes at microwave frequencies we therefore modulate an effective impedance. This is achieved by varying the parameters of either a capacitive or an inductive element in time. Although there have been early proposals for fast time-varying capacitances [31], it now is considered to be more convenient to make use of dissipationless Josephson junctions for this purpose. In a regime in which the current $I$ flowing through a Josephson junction is much smaller than its critical current $I_{C} \equiv 2 e E_{J} / \hbar$ its associated inductance is approximately $L \approx L_{J}\left(1+\frac{1}{6}\left(I(t) / I_{C}\right)^{2}\right)$. Applying an $A C$ current through the junction using appropriate microwave drive fields therefore leads to the desired time-varying impedance. Because of the proportionality of the inductance $L$ to the square of the current $I^{2}(t)$, such a drive results in a four-wave mixing process [32].

The effective impedance can alternatively be modulated by varying the magnetic flux threading a superconducting quantum interference device (SQUID) loop [5] such that the effective inductance is approximately modulated proportionally to the AC current $I(t)$ flowing in the loop, $L \approx L_{J}\left(1+I(t) / I_{0}\right)$. The quantity $I_{0}$ in this expression depends on the DC flux bias point of the SQUID loop. Since the relation between current and inductance is in this case linear, the magnetic flux drive results in a three-wave mixing process [33].

In order to enhance parametric amplification in a well-controlled frequency band while suppressing it for frequencies out of this band, the modulated Josephson inductance is frequently integrated into a microwave frequency resonator. This is the simplest way to control the band in which parametric amplification occurs. A number of variations of this basic idea are now explored. The circuit design has recently been modified to achieve a spatial separation of signal and idler modes $[9,21,34-36]$ and to build traveling wave amplifiers, in which a field is amplified while propagating in forward direction coaxially with a pump field [37, 38]. Various drive mechanisms ranging from single and double pumps [8] to magnetic flux drives [5, 39, 40] have been explored. Being aware of this variety of possible approaches, we focus here on a single mode (degenerate) parametric amplifier driven with one pump tone close to its resonance frequency.

\subsection{Circuit QED implementation of a parametric amplifier}

The JPA essentially is a weakly nonlinear oscillator, in which the nonlinearity is provided by Josephon tunnel junctions. In practice, this is typically realized either as a transmission line resonator shunted by a SQUID [7, 12,13], see Figure 1(a), or as a lumped element nonlinear oscillator [11]. The use of a SQUID instead of single tunnel junction guarantees tunability of the resonance frequency. Since resonator-based parametric amplifiers provide amplification in a narrow band only, tunability is highly desirable to match the band of amplification with the frequency of the signal to be amplified.

The relevant part of the Hamiltonian which describes the parametric amplifier considered here can be written as

$$
H_{\mathrm{JPA}}=\hbar \tilde{\omega}_{0} A^{\dagger} A+\hbar \frac{K}{2}\left(A^{\dagger}\right)^{2} A^{2}
$$



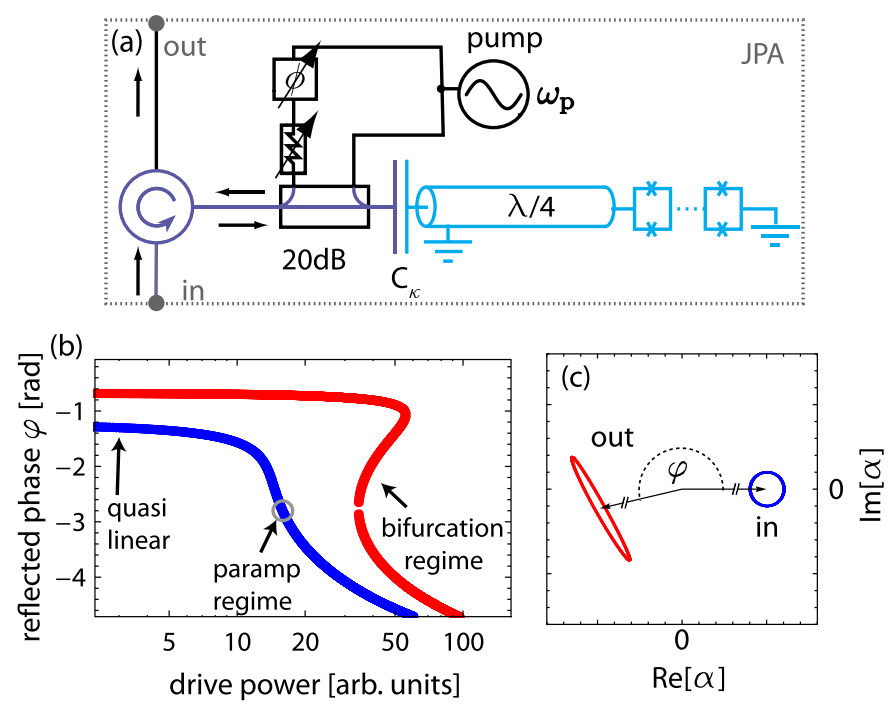

Figure 1 Schematic and operation of the parametric amplifier. (a) Circuit diagram of a transmission line resonator based parametric amplifier. The resonator is coupled with capacitance $C_{\kappa}$ to a transmission line where input and output modes are spatially separated using a circulator. A $20 \mathrm{~dB}$ directional coupler between the $\lambda / 4$-resonator and the circulator is used to apply the pump field required for modulating the SQUID inductance. The second port of the directional coupler can be used to interferometrically cancel out the pump tone reflected from the sample. (b) Phase of the reflected probe signal vs. drive power for two characteristic drive frequencies below (blue) and above (red) the bifurcation threshold. (c) Illustration of the nonlinear oscillator response in the quadrature plane. The blue circle represents various input fields $\alpha_{\text {in }}$ close to the one indicated by the gray circle in (b). Due to the nonlinear response of the resonator they are transformed into output fields $\alpha_{\text {out }}$ indicated by the red ellipse.

where $A$ labels the annihilation operator of the intra-resonator field. Expressions for the resonance frequency $\tilde{\omega}_{0} / 2 \pi$ and the effective Kerr nonlinearity $K$ are derived in Section 4 based on the full circuit model. In the following section we analytically study the dynamics of this system using the input-output formalism. Before presenting the mathematical derivations, we qualitatively describe different dynamical regimes of this nonlinear oscillator and explain the mechanism which leads to amplification.

If we assume for the moment that the JPA has no internal losses, all the incident power is reflected from the resonator and the classical response (i.e. reflection coefficient) is completely specified by the phase $\varphi$ of the reflected field. In contrast to a linear system, where $\varphi$ only depends on the frequency $\omega / 2 \pi$, it also depends on the power of the probe field in the case of a nonlinear oscillator. In Figure 1(b), the theoretically expected value of $\varphi$ is plotted as a function of the probe amplitude for two characteristic drive frequencies. While the phase is constant for low drive powers (quasi-linear response), the phase changes significantly for increased drive power. Depending on the probe frequency we either find a bistable regime where two stable solutions exist $[41,42]$ or a regime where the phase has a unique solution (red and blue data sets in Figure 1(b)). In both cases the phase significantly depends on the input power. The bistable response can for example be used to realize a bifurcation amplifier $[43,44]$ and for nonlinear dispersive readout [45], which has been intensely studied in the context of circuit QED.

Since we are particularly interested in linear amplification the following discussion is focused on the regime, in which the response has a unique solution (blue data set). The mechanism of amplification can be understood qualitatively in the following way. If we 
(a) "signal" "pump"

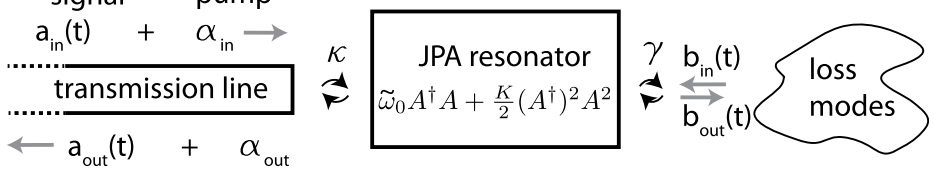

(b)

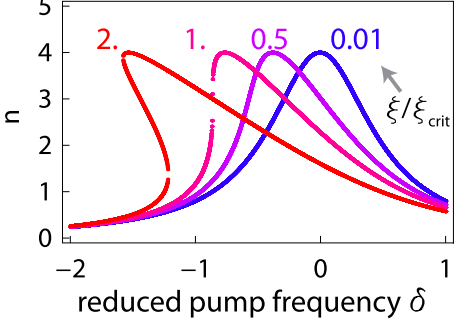

(c)

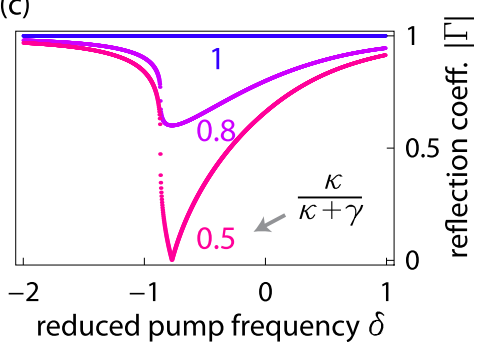

Figure 2 Input-output model. (a) Schematic of the input-output model used for calculating the response of the parametric amplifier in the presence of additional loss modes. (b) Normalized pump field photon number $n$ in the resonator as a function of reduced pump frequency $\delta$ for effective drive strengths $\xi / \xi_{\text {crit }}=0.01,0.5,1,2$, where $\xi_{\text {crit }}=-1 / \sqrt{27}$. (c) Absolute value of the reflection coefficient $|\Gamma|$ for different coupling ratios $\kappa /(\kappa+\gamma)=1,0.8,0.5$

imagine that the device is constantly driven at a frequency and power at which the reflected phase $\varphi$ depends sensitively on power (see gray circle in Figure 1(b)), the system will strongly react to small perturbations. Such perturbations, which could be caused by an additional small signal field for example, are therefore translated into a large change of the output field.

We illustrate this process leading to amplification by plotting the resonator response for input fields $\alpha_{\text {in }}$ with slightly varying amplitude and phase. In Figure 1(c) we indicate the input fields by a blue circle around the mean value (arrow). The small differences in amplitude of the input field translate into large changes in $\varphi$ of the output field $\alpha_{\text {out }}$ (red ellipse). If we interpret the arrow in Figure 1(c) as a constant pump field and its difference to the points on the blue circle as an additional signal, the signal is either amplified or deamplified depending on its phase relative to the pump.

The mechanism of amplification can thus be understood intuitively by considering the nonlinear response to a monochromatic drive field. In order to characterize the exact behavior of input fields with finite bandwidth we analyze the response in more detail below.

\section{Input-output relations for the parametric amplifier}

\subsection{Classical nonlinear response}

Here, we employ the input-output formalism $[46,47]$ to calculate the nonlinear resonator response discussed qualitatively in the previous section. The derivation presented here is inspired by Ref. [1]. A schematic of the input-output model is shown in Figure 2. The nonlinear resonator is coupled with rate $\kappa$ to a transmission line, through which the pump and signal fields propagate. Based on this model and the Hamiltonian in Eq. (1) we obtain the following equation of motion for the intra-resonator field

$$
\dot{A}=-i \tilde{\omega}_{0} A-i K A^{\dagger} A A-\frac{\kappa+\gamma}{2} A+\sqrt{\kappa} A_{\text {in }}(t)+\sqrt{\gamma} b_{\text {in }}(t) .
$$


In addition to the coupling to transmission line modes $A_{\text {in }}(t)$ with rate $\kappa$ we account for potential radiation loss mechanisms by introducing the coupling to modes $b_{\text {in }}(t)$ with loss rate $\gamma$, compare Figure 2(a). A boundary condition equivalent to

$$
A_{\text {out }}(t)=\sqrt{\kappa} A(t)-A_{\text {in }}(t)
$$

also holds for the loss modes. When operating the device as a parametric amplifier, the input field $A_{\text {in }}$ is typically a sum of a strong coherent pump field and an additional weak signal field. Since this signal carries at least the vacuum noise, it is treated as a quantum field. In this formalism this particular situation is accounted for by decomposing each field mode into a sum of a classical part and a quantum part

$$
\begin{aligned}
& A_{\text {in }}(t)=\left(a_{\text {in }}(t)+\alpha_{\text {in }}\right) e^{-i \omega_{p} t}, \\
& A_{\text {out }}(t)=\left(a_{\text {out }}(t)+\alpha_{\text {out }}\right) e^{-i \omega_{p} t}, \\
& A(t)=(a(t)+\alpha) e^{-i \omega_{p} t},
\end{aligned}
$$

where $\alpha, \alpha_{\text {in }}, \alpha_{\text {out }}$ represent the classical parts of the field which are associated with the pump, while $a, a_{\text {in }}, a_{\text {out }}$ account for the quantum signal fields. Since all $\alpha$ 's are complex numbers the modes $a$ satisfy the same bosonic commutation relations as modes $A$ do. By multiplying the field modes defined in Eq. (4) with the additional exponential factor $e^{-i \omega_{p} t}$, one works in a frame rotating at the pump frequency $\omega_{p}$. The strategy is to first solve the classical response for the pump field $\alpha$ exactly and then linearize the equation of motion for the weak quantum field $a$ in the presence of the pump. Finally, we derive a scattering relation between input modes $a_{\text {in }}$ and reflected modes $a_{\text {out }}$.

The steady state solution for the coherent pump field is determined by

$$
\left(i\left(\tilde{\omega}_{0}-\omega_{p}\right)+\frac{\kappa+\gamma}{2}\right) \alpha+i K \alpha^{2} \alpha^{*}=\sqrt{\kappa} \alpha_{\mathrm{in}}
$$

which follows immediately by substituting Eq. (4) into Eq. (2) and collecting only the cnumber terms. By multiplying both sides with their complex conjugate we get to the equation

$$
\frac{\kappa}{(\kappa+\gamma)^{2}}\left|\alpha_{\mathrm{in}}\right|^{2}=\left(\left(\frac{\omega_{p}-\tilde{\omega}_{0}}{\kappa+\gamma}\right)^{2}+\frac{1}{4}\right)|\alpha|^{2}-\frac{2\left(\omega_{p}-\tilde{\omega}_{0}\right) K}{(\kappa+\gamma)^{2}}|\alpha|^{4}+\left(\frac{K}{\kappa+\gamma}\right)^{2}|\alpha|^{6},
$$

which determines the average number of pump photons $|\alpha|^{2}$ in the resonator. Eq. (6) reduces to

$$
1=\left(\delta^{2}+\frac{1}{4}\right) n-2 \delta \xi n^{2}+\xi^{2} n^{3}
$$

by defining the scale invariant quantities

$$
\delta \equiv \frac{\omega_{p}-\tilde{\omega}_{0}}{\kappa+\gamma}, \quad \tilde{\alpha}_{\mathrm{in}} \equiv \frac{\sqrt{\kappa} \alpha_{\mathrm{in}}}{\kappa+\gamma}, \quad \xi \equiv \frac{\left|\tilde{\alpha}_{\mathrm{in}}\right|^{2} K}{\kappa+\gamma}, \quad n \equiv \frac{\left|\alpha^{2}\right|}{\left|\tilde{\alpha}_{\text {in }}\right|^{2}} .
$$

$\delta$ is the detuning between pump and resonator frequency in units of the total resonator linewidth, $\tilde{\alpha}_{\text {in }}$ is the dimensionless drive amplitude, and $\xi$ is the product of drive power 
and nonlinearity, also expressed in dimensionless units. Finally, $n$ is the mean number of pump photons in the resonator relative to the incident pump power. As an important consequence, we notice from Eq. (8) that only the product of drive power and nonlinearity determines the dynamics but not each quantity itself. Therefore, a small nonlinearity can at least in principle be compensated by increasing the drive power. Properties such as the gain-bandwidth product are therefore independent of the strength of the nonlinearity as long as the pump power is much larger than the power of amplified fluctuations. Furthermore, the solutions of Eq. (7) for negative $\xi$ values are identical to those for positive $\xi$ up to a sign change in $\delta$. Since $\xi$ is negative for the Josephson parametric amplifier, we focus on this particular case.

Equation (7) is a cubic equation in $n$ and can therefore be solved analytically. We do not present the lengthy solutions here explicitly, but assume in the following that we have an explicit analytical expression for $n$ in terms of $\delta$ and $\xi$. In Figure 2(b) we plot $n$ for various parameters $\xi$ as a function of $\delta$. At the critical value $\xi_{\text {crit }}=-1 / \sqrt{27}$ the derivative $\partial n / \partial \delta$ diverges and thus the response of the parametric amplifier becomes extremely sensitive to small changes. For even stronger effective drive powers $\xi / \xi_{\text {crit }}>1$ the cubic Eq. (7) has three real solutions. The solutions for the high and low photon numbers are stable, while the intermediate one is unstable. The system bifurcates in this regime as mentioned earlier. The critical detuning below which the system becomes bistable is $\delta_{\text {crit }}=-\sqrt{3} / 2$. The critical point $\left(\xi_{\text {crit }}, \delta_{\text {crit }}\right)$ is the one at which both $\partial \delta / \partial n$ and $\partial^{2} \delta / \partial^{2} n$ vanish. In scale invariant units the maximal value of $n$ is 4 , which is reached at the detuning $\delta=4 \xi$.

Experimentally, the system parameters are characterized by measuring the complex reflection coefficient $\Gamma \equiv \alpha_{\text {out }} / \alpha_{\text {in }}$. Based on the input-output relation $\alpha_{\text {out }}=\sqrt{\kappa} \alpha-\alpha_{\text {in }}$ and Eq. (5) we evaluate this reflection coefficient as

$$
\Gamma=\frac{\kappa}{\kappa+\gamma} \frac{1}{\frac{1}{2}-i \delta+i \xi n}-1
$$

In Figure 2(c) we plot the absolute value of the reflection coefficient at $\xi=\xi_{\text {crit }}$ for various loss rates $\gamma$. For vanishing losses $\gamma=0$ all the incident drive power is reflected from the device and $|\Gamma|=1$. Note that also in this case the resonance is clearly visible in the phase of the reflected signal (not shown here). When the loss rate $\gamma$ becomes similar to the external coupling rate $\kappa$ part of the radiation is dissipated into the loss modes. In the case of critical coupling $\gamma=\kappa$ all the coherent power is transmitted into the loss modes at resonance. This is equivalent to the case of a symmetrically coupled $\lambda / 2$ resonator, for which the transmission coefficient is one at resonance [48].

\subsection{Linearized response for weak (quantum) signal fields}

Under the assumption that the photon flux associated with the signal $\left\langle a_{\mathrm{in}}^{\dagger} a_{\mathrm{in}}\right\rangle$ is much smaller than the photon flux of the pump field $\left|\alpha_{\text {in }}\right|^{2}$, we can drop terms such as $K a^{\dagger} a \alpha$, because they are small compared to the leading terms $K a^{\dagger} \alpha^{2}$ and $K a|\alpha|^{2}$. By neglecting these terms we obtain a linearized equation of motion for $a$ in the presence of the pump field. In order to preserve the validity of this approximation even for larger input signals, the amplitude $\alpha$ of the pump field needs to be increased. Experimentally, this can be achieved by reducing the strength of the nonlinearity $K$ as discussed in Section 3.3 in more detail. 
Substituting Eq. (4) into Eq. (2) and keeping only terms which are linear in $a$ one finds

$$
\dot{a}(t)=i\left(\omega_{p}-\tilde{\omega}_{0}-2 K|\alpha|^{2}+i \frac{\kappa+\gamma}{2}\right) a(t)-i K \alpha^{2} a^{\dagger}(t)+\sqrt{\kappa} a_{\mathrm{in}}(t)+\sqrt{\gamma} b_{\mathrm{in}}(t) .
$$

Since Eq. (10) is linear, we can solve it by decomposing all modes into their Fourier components

$$
a(t) \equiv \frac{\kappa+\gamma}{\sqrt{2 \pi}} \int_{-\infty}^{\infty} \mathrm{d} \Delta e^{-i \Delta(\kappa+\gamma) t} a_{\Delta}
$$

and equivalently for $a_{\mathrm{in}, \Delta}$ and $b_{\mathrm{in}, \Delta}$. Note that the detuning $\Delta$ between signal frequencies and the pump frequency, is expressed here in units of the linewidth $\kappa+\gamma$. Substituting the Fourier decompositions into Eq. (10) and comparing the coefficients of different harmonics, results in

$$
0=\left(i(\delta-2 \xi n+\Delta)-\frac{1}{2}\right) a_{\Delta}-i \xi n e^{2 i \phi} a_{-\Delta}^{\dagger}+\tilde{c}_{\mathrm{in}, \Delta},
$$

where $\tilde{c}_{\mathrm{in}, \Delta} \equiv\left(\sqrt{\kappa} a_{\mathrm{in}, \Delta}+\sqrt{\gamma} b_{\mathrm{in}, \Delta}\right) /(\kappa+\gamma)$ is the sum of all field modes incident on the resonator. Furthermore, in Eq. (12) $\phi$ is the phase of the intra-resonator pump field, defined by $\alpha=|\alpha| e^{i \phi}$. The fact that Eq. (12) couples modes $a_{\Delta}$ and $a_{-\Delta}^{\dagger}$ can be interpreted as a wave mixing process. In order to express $a_{\Delta}$ in terms of the input fields $c_{\mathrm{in}, \Delta}$, Eq. (12) is rewritten as a matrix equation

$$
\left(\begin{array}{c}
\tilde{c}_{\mathrm{in}, \Delta} \\
\tilde{c}_{\mathrm{in},-\Delta}^{\dagger}
\end{array}\right)=\left(\begin{array}{cc}
i(-\delta+2 \xi n-\Delta)+\frac{1}{2} & i \xi n e^{i 2 \phi} \\
-i \xi n e^{-i 2 \phi} & i(\delta-2 \xi n-\Delta)+\frac{1}{2}
\end{array}\right)\left(\begin{array}{c}
a_{\Delta} \\
a_{-\Delta}^{\dagger}
\end{array}\right) .
$$

By inverting the matrix on the right hand side, the quantum part of the intra-resonator field $a_{\Delta}$ is expressed in terms of the incoming field $\tilde{c}_{\mathrm{in}, \Delta}$

$$
a_{\Delta}=\frac{i(\delta-2 \xi n-\Delta)+\frac{1}{2}}{\left(i \Delta-\lambda_{-}\right)\left(i \Delta-\lambda_{+}\right)} \tilde{c}_{\mathrm{in}, \Delta}+\frac{-i \xi n e^{2 i \phi}}{\left(i \Delta-\lambda_{-}\right)\left(i \Delta-\lambda_{+}\right)} \tilde{c}_{\mathrm{in},-\Delta}^{\dagger}
$$

with $\lambda_{ \pm}=\frac{1}{2} \pm \sqrt{(\xi n)^{2}-(\delta-2 \xi n)^{2}}$. Using Eq. (3), the final transformation between input and output modes is

$$
\begin{aligned}
& a_{\mathrm{out}, \Delta}=g_{S, \Delta} a_{\mathrm{in}, \Delta}+g_{I, \Delta} a_{\mathrm{in},-\Delta}^{\dagger}+\sqrt{\frac{\gamma}{\kappa}}\left(g_{S, \Delta}+1\right) b_{\mathrm{in}, \Delta}+\sqrt{\frac{\gamma}{\kappa}} g_{I, \Delta} b_{\mathrm{in},-\Delta}^{\dagger} \\
& \stackrel{\gamma / \kappa \rightarrow 0}{=} g_{S, \Delta} a_{\mathrm{in}, \Delta}+g_{I, \Delta} a_{\mathrm{in},-\Delta}^{\dagger},
\end{aligned}
$$

with

$$
g_{S, \Delta}=-1+\frac{\kappa}{\kappa+\gamma} \frac{i(\delta-2 \xi n-\Delta)+\frac{1}{2}}{\left(i \Delta-\lambda_{-}\right)\left(i \Delta-\lambda_{+}\right)}
$$

and

$$
g_{I, \Delta}=\frac{\kappa}{\kappa+\gamma} \frac{-i \xi n e^{2 i \phi}}{\left(i \Delta-\lambda_{-}\right)\left(i \Delta-\lambda_{+}\right)} .
$$



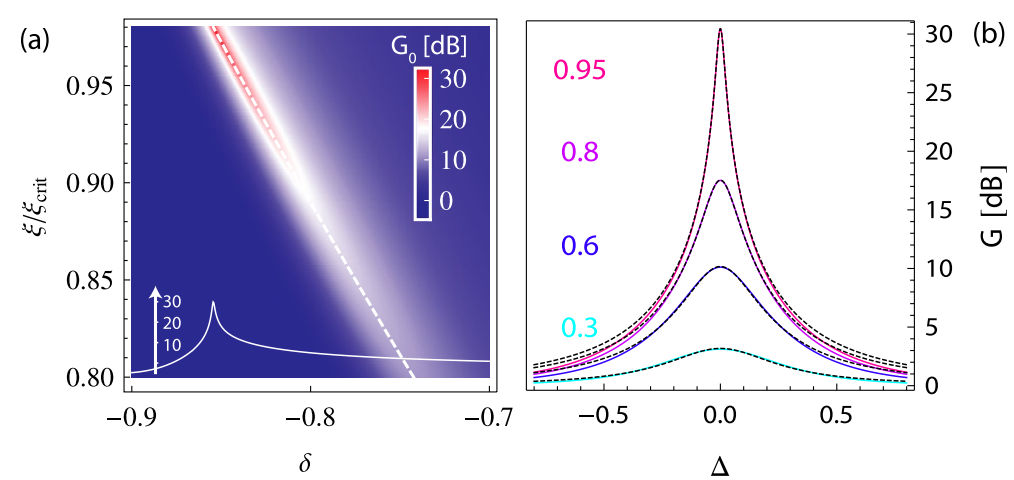

Figure 3 Parametric amplifier gain. (a) $G_{\Delta}=\left|g_{S, \Delta}\right|^{2}$ vs. pump tone detuning $\delta$ and drive strength $\xi$ at zero signal detuning $\Delta$ and for $\kappa=\gamma$. For increasing drive strength $\xi$ the detuning for maximum gain is indicated by the dashed white line. A cut through the data for the highest value $\xi=0.98 \xi_{\text {crit }}$ is shown as the solid white line in the bottom part. (b) Gain as a function of signal detuning $\Delta$ for the indicated drives strengths $\xi / \xi_{\text {crit }}$ and optimal pump detuning. The exact gain curves (solid lines) are well approximated by Lorentzian lines (black dashed lines).

Eq. (15b) is the central result of this calculation. The output field at detuning $\Delta$ from the pump frequency is a sum of the input fields at frequencies $\Delta$ and $-\Delta$ multiplied with the signal gain factor $g_{S, \Delta}$ and the idler gain factor $g_{I, \Delta}$, respectively. The additional noise contributions introduced via the loss modes $b_{\mathrm{in}, \Delta}$ vanish in the limit $\gamma / \kappa \rightarrow 0$. In the ideal case $\gamma=0$, the coefficients $g_{S, \Delta}$ and $g_{I, \Delta}$ satisfy the relation

$$
G_{\Delta} \equiv\left|g_{S, \Delta}\right|^{2}=\left|g_{I, \Delta}\right|^{2}+1
$$

and Eq. (15b) is identical to a two-mode squeezing transformation $[19,49]$ with gain $G_{\Delta}$. The two-mode squeezing transformation describes a linear amplifier in its minimal form (compare Ref. [50]), of which we discuss characteristic properties in the following section.

\subsection{Gain, bandwidth, noise and dynamic range}

For simplicity we consider the case of no losses $\gamma=0$, for which the parametric amplifier response is described by Eq. (15b). An incoming signal at detuning $\Delta$ is thus amplified by the power gain $G_{\Delta}=\left|g_{S, \Delta}\right|^{2}$ and mixed with the frequency components at the opposite detuning from the pump. Characteristic properties of the parametric amplifier, such as the maximal gain and the bandwidth, are thus encoded in the quantity $g_{S, \Delta}$ as a function of pump-resonator detuning $\delta$, effective drive strength $\xi$ and detuning between signal and pump $\Delta$.

In Figure 3(a) we plot the gain $G_{0}$ for zero signal detuning $\Delta=0$ as a function of $\delta$ and $\xi$. We find that the maximal gain increases with increasing drive strength $\xi$ while the optimal value for $\delta$ at which this gain is reached, shifts approximately linearly with increasing $\xi$. The optimal values for $\delta$ are indicated as a dashed white line in Figure 3(a). Mathematically, the gain diverges when $\xi$ approaches the critical value $\xi_{\text {crit }}$. In practice, the gain is limited to finite values due to the breakdown of the stiff pump approximation (see discussion below).

By changing the pump parameters $\xi$ and $\delta$ we can adjust the gain $G_{0}$ to a desirable value, which is typically about $20 \mathrm{~dB}$. Note that the gain can take values smaller than one, in the 
presence of finite internal losses $\gamma>0$. Once the pump parameters are fixed we characterize the bandwidth of the amplifier by analyzing the gain as a function of the signal detuning $\Delta$. In Figure 3(b) we plot the gain as a function of $\Delta$ for the indicated values of $\xi / \xi_{\text {crit }}$ and the corresponding optimal pump detunings $\delta$ (compare dashed white line in (a)). The gain curves are well approximated by Lorentzian lines as indicated by the dashed black lines in Figure 3(b). When the gain is increased, the band of amplification becomes narrower. This is quantitatively expressed by the gain-bandwidth relation $\sqrt{G_{0}} B \approx 1$, where $B$ is the detuning $\Delta$ for which the gain reaches half of its maximal value. This gain-bandwidth relation follows from the Lorentzian approximation of the gain curves shown as black dashed lines in Figure 3(b)) and holds for gain values above a few dB. Remember that $\Delta$ is defined in units of the resonator linewidth $\kappa+\gamma$, which means that the amplifier bandwidth equals approximately the resonator linewidth divided by the square root of the gain.

When operating the JPA, we also have to understand its behavior in terms of added noise. In the ideal case with zero loss rate $(\gamma=0)$, the input-output relation of the parametric amplifier in Eq. (15b) has the minimal form of a scattering mode amplifier [19]. The amplification process reaches the vacuum limit as long as the input modes are cooled into the vacuum. In practice, however, the device may have finite loss $\gamma$ which increases the effectively added noise by a factor of $(\kappa+\gamma) / \kappa$. This is due to the additional amplified noise, which originates from the modes $b_{\mathrm{in}, \Delta}$ and contributes to the output field $a_{\mathrm{out}, \Delta}$ (compare Eq. (15a)). Another potential source of noise is related to the stability of the resonance frequency of the parametric amplifier. Magnetic flux noise in the SQUID loop may lead to a fluctuating resonance frequency and thus a fluctuating effective gain.

In the derivation made in the previous sections we have assumed that the solution of the classical drive field is unaffected by the presence of additional signal and quantum fluctuations at the input. This is known as the stiff pump approximation [8], which assumes that the pump power at the output is equal to the pump power at the input of the JPA. This is of course an approximation, since the pump field provides the energy which is necessary for amplifying the input signal. The stiff pump approximation is valid as long as the pump power is significantly larger than the total output power of all amplified (quantum) signal and vacuum fields. In order to quantitatively analyze the pump depletion due to the presence of amplified fields we add the terms $2 i K\left\langle a^{\dagger} a\right\rangle \alpha$ and $i K\left\langle a^{2}\right\rangle \alpha^{*}$ to the left hand side of Eq. (5) and solve Eq. (5) and Eq. (10) self-consistently. This mean-field approach is similar to the one used in Ref. [8]. For our calculation we model the incoming signal field, which is to be amplified, as white noise with average photon number $n_{\text {th }}$ per unit time and bandwidth. Based on this model we find that the gain decreases when the signal strength exceeds a certain value, see Figure 4(a). The number of input photons $n_{\text {th }}$ at which this happens becomes smaller with decreasing ratio $\kappa /|K|$. This is expected because the pump power close to the bifurcation point is proportional to $\kappa /|K|$ and provides the energy required for amplification. For small values $\kappa /|K|$ the gain is reduced even for $n_{\text {th }}=0$ due to the amplification of vacuum fluctuations (see blue data points in Figure 4(a)). As a measure of the dynamic range we specify the $1 \mathrm{~dB}$ compression point of the JPA, i.e. the value $n_{1 \mathrm{~dB}}$ of input photons $n_{\text {th }}$ at which the JPA gain $G_{0}$ decreases by $1 \mathrm{~dB}$ compared to the stiff pump approximated gain value. As shown in Figure 4(b), the $1 \mathrm{~dB}$ compression point increases proportionally to $\kappa /|K|$ in the limit of $n_{1 \mathrm{~dB}} \gg 1$. The presence of constant vacuum fluctuations leads to a gain compression by more than $1 \mathrm{~dB}$ even for $n_{\text {th }}=0$ when $\kappa /|K|$ becomes smaller. We can qualitatively explain this behavior by comparing the pump 
(a)

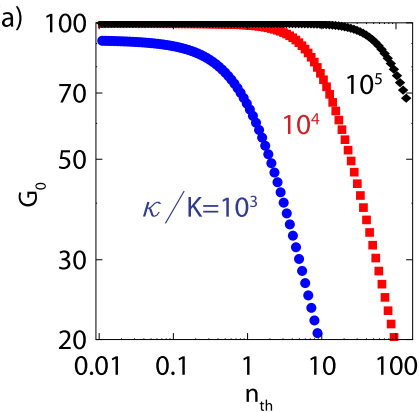

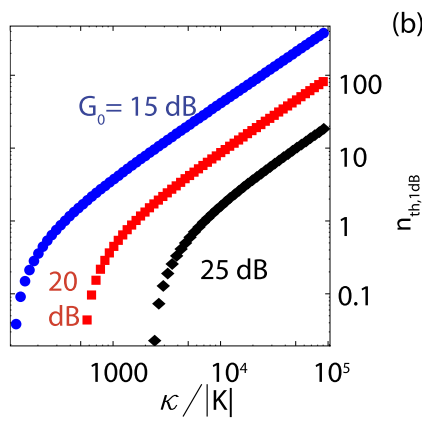

(b)

\section{$\check{c}^{\overline{5}}$}

Figure 4 Dynamic range of the JPA. (a) Gain $G_{0}$ as a function of the photon number $n_{\text {th }}$ of the input signal field for the three indicated ratios $\kappa /|K|$. (b) $1 \mathrm{~dB}$ compression point as a function of $\kappa /|K|$ for various gain values $G_{0}$.

power $P_{p}=\hbar \omega_{p}\left|\alpha_{\text {in }}\right|^{2}$ with the power of amplified signal and vacuum fields

$$
P_{\text {out }} \stackrel{\gamma=0}{=} \hbar \omega_{p} \kappa\left(2 n_{\mathrm{th}}+1\right) \int \frac{\mathrm{d} \Delta}{2 \pi}\left(G_{\Delta}-1\right) .
$$

Making use of the gain-bandwidth relation we find the following scaling of the ratio between the two powers

$$
\frac{P_{\text {out }}}{P_{p}} \propto\left(2 n_{\mathrm{th}}+1\right) \frac{|K|}{\kappa} \sqrt{G_{0}} .
$$

The results shown in Figure 4(b) together with Eq. (20) indicate that the validity of the stiff pump approximation is essentially determined by the ratio $P_{\text {out }} / P_{p}$. The calculations furthermore show that the dynamic range can be increased by reducing the ratio $|K| / \kappa$ of the JPA, which seems to be the case also for flux driven parametric amplifiers [40]. In Section 5 we discuss how to achieve small nonlinearities by making use of multiple SQUIDs connected in series.

\section{Effective system parameters from distributed circuit model}

In the previous section we have analyzed the model of a nonlinear resonator with resonance frequency $\tilde{\omega}_{0}$, Kerr nonlinearity $K$ and decay rate $\kappa$. Here, we explicitly derive this effective Hamiltonian from the full circuit model of a $\lambda / 4$ - transmission line resonator, which is terminated by a SQUID loop at the short-circuited end and coupled capacitively to a transmission line, see Figure $1(\mathrm{a})$. These calculations allow us to determine $\tilde{\omega}_{0}, K, \kappa$ from the distributed circuit parameters and give insight into potential limitations of the effective model. We also compare the obtained parameter relations with those of a lumped element parametric amplifier.

\subsection{Resonator mode structure in the linear regime}

In order to find the normal mode structure of the system, we first neglect its capacitive coupling to the transmission line as indicated in Figure 5. The derivation is similar to the ones in Refs. [40,51,52]. Dissipation effects due the environment are discussed in Section 4.3. 


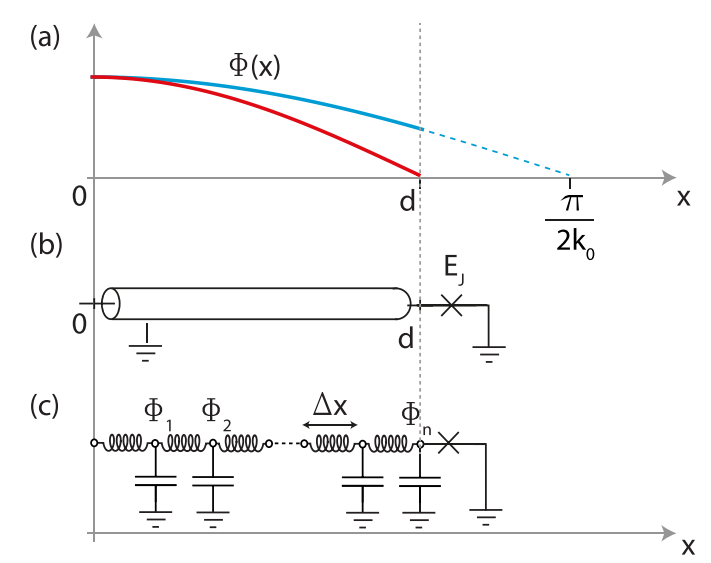

Figure 5 Circuit model. (a) Distribution of the magnetic flux field $\Phi(x)$ along the $\lambda / 4$-resonator for the fundamental resonator mode $j=0$, without (red) and with (blue) a Jospehson junction. The additional Josephson inductance changes the boundary condition such that neither the current nor the voltage is zero at position $x=d$. The resulting increase in the effective wavelength $\pi /\left(2 k_{0}\right)$ is indicated by the dashed blue line. (b) Transmission line resonator of length $d$ with a Josephson junction at the grounded end. (c) Lumped element representation with indicated discretized magnetic flux field $\Phi_{j}$ as used in Eq. (21).

The total Lagrangian of the system in the magnetic flux field $\Phi(x)$ has a transmission line part and a term which describes the SQUID at position $x=d$ (Figure 5).

$$
\mathcal{L}=\int_{0}^{d} \mathrm{~d} x\left\{\frac{c}{2}\left(\partial_{t} \Phi(x)\right)^{2}-\frac{1}{2 l}\left(\partial_{x} \Phi(x)\right)^{2}\right\}+E_{J} \cos \left(\frac{\Phi(d)}{\varphi_{0}}\right),
$$

with the reduced flux quantum $\varphi_{0}=\hbar / 2 e$. Since we work in a limit in which the plasma frequency of the SQUID is much larger than the resonance frequencies of interest, we neglect the self-capacitance of the SQUID. Note that Ref. [52] provides a detailed study of the effect of the self-capacitance in various parameter regimes. We furthermore describe the SQUID as a single junction with tunable effective Josephson energy $E_{J}$.

We first investigate the linear regime of the system, in which the cosine potential of the SQUID is approximated as a quadratic potential.

$$
E_{\mathrm{J}} \cos \left(\frac{\Phi(d)}{\varphi_{0}}\right) \approx \text { const }-\frac{1}{2}\left(\frac{\Phi(d)}{\varphi_{0}}\right)^{2}
$$

Due to the spatial derivative in the Lagrangian in Eq. (21) all local fields in the chain are coupled to their nearest neighbors and the normal mode structure is found by solving the Euler-Lagrange equation $\partial_{t}(\delta \mathcal{L} / \delta \dot{\Phi})-\delta \mathcal{L} / \delta \Phi=0$ of the transmission line resonator. This results in the wave equation

$$
v^{2} \partial_{x}^{2} \Phi(x)-\partial_{t}^{2} \Phi(x)=0
$$

with the phase velocity $v=1 / \sqrt{c l}$, of which the general solution can be written as a sum of normal modes

$$
\Phi(x)=\sum_{j=0}^{\infty} \phi_{j} \cos \left(k_{j} x\right) .
$$


The valid wavevectors $k_{j}$ are determined by the boundary conditions at the two ends of the $\lambda / 4$ transmission line. The open end at $x=0$ requires that the current $\partial_{x} \Phi(x) / l$ vanishes, which is implicitly satisfied by choosing the cosine ansatz in Eq. (24). On the shorted end the boundary condition is modified by the presence of the Josephson junction. In order to determine this boundary condition, we evaluate the Euler-Lagrange equation at position $x=d$. For this purpose it is convenient to write the Lagrangian in a discretized form, see Figure 5(c) and compare Ref. [51]:

$$
\mathcal{L}=\lim _{n \rightarrow \infty} \sum_{j=1}^{n} \Delta x\left\{\frac{c}{2}\left(\partial_{t} \Phi_{j}\right)^{2}-\frac{1}{l} \frac{\left(\Phi_{j}-\Phi_{j-1}\right)^{2}}{\Delta x^{2}}\right\}-\frac{1}{2} E_{\mathrm{J}}\left(\frac{\Phi_{n}}{\varphi_{0}}\right)^{2}
$$

where $\Phi_{n}=\Phi(x=d)$ and $\Delta x=d / n$. Evaluating $\partial_{t}\left(\partial \mathcal{L} / \partial \dot{\Phi}_{n}\right)-\partial \mathcal{L} / \partial \Phi_{n}=0$ leads to the equation

$$
\frac{1}{l} \partial_{x} \Phi(d)+E_{\mathrm{J}} \frac{\Phi(d)}{\varphi_{0}^{2}}=0
$$

Substituting the ansatz (24) into Eq. (26) and comparing the resulting coefficients of the independent variables $\phi_{j}$, results in the transcendental equation

$$
k_{j} d \tan \left(k_{j} d\right)=l d \frac{E_{\mathrm{J}}}{\varphi_{0}^{2}} \equiv \frac{l d}{L_{\mathrm{J}}} .
$$

Here, we have defined the Josephson inductance $L_{J}=\varphi_{0}^{2} / E_{\mathrm{J}}$. The infinite set of solutions $k_{j}$ of this equation determines the normal modes structure of the system in the linear regime. In the limit in which the SQUID inductance $L_{J}$ vanishes, Eq. (27) is solved by the poles of $\tan \left(k_{j} d\right)$, and we recover the normal modes of the $\lambda / 4$ resonator

$$
k_{j}^{(0)} d=\frac{\pi}{2}(1+2 j) \quad \text { with } j \in\{0,1,2,3, \ldots\} .
$$

As a first order correction to this result in the limit of $L_{\mathrm{J}} / l d \ll 1$, we expand Eq. (27) to first order in $\left(k_{j}^{(0)}-k_{j}\right) d$ and find $k_{j} L_{\mathrm{J}} / l d=\left(k^{(0)}-k_{j}\right)$ or equivalently

$$
k_{j} \approx \frac{k_{j}^{(0)}}{1+L_{\mathrm{J}} / l d} .
$$

For the fundamental mode with $j=0$ this linearized approximation is typically accurate even for inductance ratios up to $L_{\mathrm{J}} / l d \approx 0.5$, whereas for the higher harmonic modes the linearized equation breaks down for much smaller values of $L_{\mathrm{J}} / l d$. A comparison between the exact solution based on Eq. (27) and the approximate solution in Eq. (29) is shown in Figure 6 for the first three resonant modes. When higher harmonics are expected to be relevant one should solve Eq. (27) numerically in order to determine the exact wave numbers $k_{j}$.

\subsection{Kerr nonlinear terms and effective Hamiltonian}

Using the normal mode decomposition in Eq. (24) we reexpress the Lagrangian in Eq. (21) as a sum of oscillators which are only coupled via the boundary condition imposed by the 
Figure 6 Resonance frequencies of the first three modes as a function of Josephson energy. The solid line results from the exact numerical solution of Eq. (27) while the dashed line shows the linearized solution in Eq. (29). The bare resonance frequency is chosen to be $7 \mathrm{GHz}$ and the impedance of the transmission line resonator $50 \Omega$

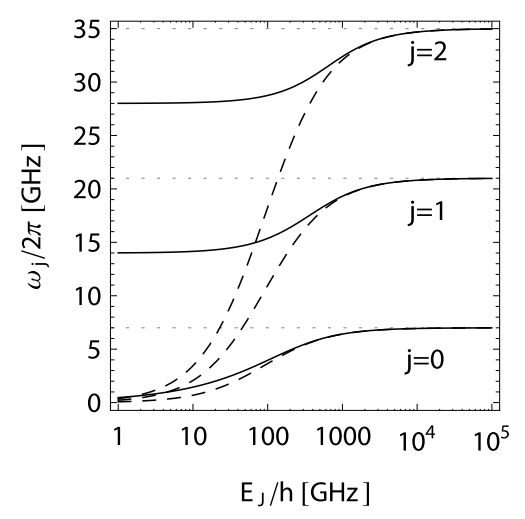

SQUID. For the purposes of parametric amplification the phase drop across the junction is desired to be small, $\Phi_{n} / \varphi_{0}<1$, i.e. the current flowing through the Josephson junction is small compared to its critical current. We can therefore expand the SQUID cosine potential and take into account only the first non-quadratic correction

$$
E_{\mathrm{J}} \cos \left(\frac{\Phi_{n}}{\varphi_{0}}\right)=\text { const }-\frac{1}{2} E_{\mathrm{J}}\left(\frac{\Phi_{n}}{\varphi_{0}}\right)^{2}+\frac{1}{24} E_{\mathrm{J}}\left(\frac{\Phi_{n}}{\varphi_{0}}\right)^{4}+\cdots
$$

In Section 5 we discuss under which circumstances such an approximation may break down. Substituting the normal mode decomposition Eq. (24) into the Taylor expansion of the Lagrangian results in

$$
\mathcal{L}=\frac{1}{2} \sum_{i=1}^{\infty}\left\{\dot{\phi}_{i} C_{i} \dot{\phi}_{i}-\phi_{i} L_{i}^{-1} \phi_{i}\right\}+\sum_{j, i, k, l=1}^{\infty} N_{i j k l} \phi_{i} \phi_{j} \phi_{k} \phi_{l}
$$

with the effective capacitances and inductances [51]

$$
\begin{gathered}
C_{i}=c \int_{0}^{d} \mathrm{~d} x \cos ^{2}\left(k_{i} x\right)=\frac{c d}{2}\left(1+\frac{\sin \left(2 k_{i} d\right)}{2 k_{i} d}\right), \\
L_{i}^{-1}=L_{\mathrm{J}}^{-1} \cos ^{2}\left(k_{i} d\right)+\frac{k_{i}^{2}}{l} \int_{0}^{d} \mathrm{~d} x \sin ^{2}\left(k_{i} x\right) \\
\stackrel{\text { Eq. }}{=}(27) \frac{\left(k_{i} d\right)^{2}}{2 l d}\left(1+\frac{\sin \left(2 k_{i} d\right)}{2 k_{i} d}\right),
\end{gathered}
$$

and the nonlinearity coefficients

$$
N_{i j k l}=\frac{1}{24} E_{\mathrm{J}} \varphi_{0}^{-4} \prod_{m \in\{i, j, k, l\}} \cos \left(k_{m} d\right) .
$$

As expected the linear part of the Lagrangian is diagonal in the normal mode basis. It describes a set of uncoupled $L C$ oscillators for which the effective resonance frequencies coincide with the product of phase velocity and wave vector $\omega_{j}=k_{j} v=1 / \sqrt{L_{j} C_{j}}$.

Based on the Lagrange function (31) we derive the Hamiltonian by introducing the conjugate charge variables $q_{i}=\delta \mathcal{L} / \delta \dot{\phi}_{i}=C_{i} \dot{\phi}_{i}$. Performing a Legendre transformation and tak- 
ing only self-interactions and two-mode interactions into account, results in the Hamiltonian

$$
\mathcal{H}=\frac{1}{2} \sum_{i=1}^{\infty}\left\{q_{i} C_{i}^{-1} q_{i}+\phi_{i} L_{i}^{-1} \phi_{i}\right\}-3 \sum_{j \neq i}^{\infty} N_{i i j j} \phi_{i}^{2} \phi_{j}^{2}-\sum_{i}^{\infty} N_{i i i i} \phi_{i}^{4} .
$$

In a quantum regime $q_{i}$ and $\phi_{i}$ are operators which satisfy the commutation relation $\left[\phi_{j}, q_{k}\right]=\delta_{k j} \hbar / i$ and it is convenient to write the Hamiltonian in terms of normal mode annihilation and creation operators [53]

$$
\phi_{j}=i \phi_{\mathrm{zpf}, j}\left(a_{j}^{\dagger}-a_{j}\right), \quad q_{j}=q_{\mathrm{zpf}, j}\left(a_{j}+a_{j}^{\dagger}\right)
$$

with $q_{\mathrm{zpf}, i}=\sqrt{\hbar \omega_{i} C_{i} / 2}$ and $\phi_{\mathrm{zpf}, i}=\sqrt{\hbar / 2 \omega_{i} C_{i}}$. The abbreviation zpf stands for zero point fluctuations. Performing a rotating wave approximation (i.e. removing all terms with an unequal number of creation and annihilation operators), and neglecting the small photon number independent frequency shifts due to the nonlinear terms (i.e. Lamb shifts) we arrive at

$$
\mathcal{H}=\sum_{i=1}^{\infty} \hbar \omega_{i} a_{i}^{\dagger} a_{i}+\hbar \frac{K_{i i}}{2} a_{i}^{\dagger} a_{i}^{\dagger} a_{i} a_{i}+\sum_{j \neq i}^{\infty} \hbar K_{i j} a_{i}^{\dagger} a_{i} a_{j}^{\dagger} a_{j}
$$

with

$$
K_{i j}=-\frac{E_{\mathrm{J}}}{2 \hbar}\left(\frac{\phi_{\mathrm{zpf}}}{\varphi_{0}}\right)^{4} \cos ^{2}\left(k_{i} d\right) \cos ^{2}\left(k_{j} d\right)
$$

The quantity $K=K_{00}$ is the Kerr nonlinearity of the fundamental mode, which is used for the parametric amplification process. The terms proportional to $K_{i j}$ with unequal $i \neq j$ are cross Kerr interaction terms which couple different modes to each other. Such an interaction can for example be used for counting the number of photons in one mode by probing another one with a coherent field [54-61], similarly to a dispersive qubit measurement. Note that the values resulting from Eq. (37) are divided by the square of the number of SQUIDs, if an array is used instead of a single SQUID, as discussed in the following section.

\subsection{Decay rate and resonance frequency correction for low $Q$ resonators}

Since the parametric amplifier bandwidth is proportional to the decay rate $\kappa$, typical devices are designed to have a low external quality factor, which is achieved by increasing the coupling capacitance $C_{k}$ between transmission line and resonator (Figure 1). The coupling of an oscillator to the environment shifts its resonance frequency $\omega_{j} \rightarrow \tilde{\omega}_{j}$ [48], which can be significant if the coupling rate is large. When designing parametric amplifier devices, it is therefore necessary to take these shifts into account. Based on the effective inductance and capacitances calculated in Eq. (32) we find

$$
\tilde{\omega}_{j}^{2} \approx \frac{\omega_{j}^{2}}{1+C_{\kappa} / C_{j}}=\frac{1}{\left(C_{j}+C_{\kappa}\right) L_{j}} \quad \text { and } \quad \kappa_{j} \approx \frac{\tilde{\omega}_{j}^{2} C_{\kappa}^{2} R}{C_{\kappa}+C_{j}}
$$

for resonance frequency and decay rate of the $j$ th mode of the parametric amplifier device. The external quality factor is given by $Q_{j} \equiv \tilde{\omega}_{j} / \kappa_{j}$. 


\subsection{Lumped element JPA}

As already mentioned in the introduction, a JPA can also be realized as a lumped element resonator by shunting a SQUID with a large capacitance $C_{J}[10,11]$. In this case the resonator is described by the transmon Hamiltonian [62], which in the deep transmon limit $E_{J} \gg E_{C} \equiv e^{2} / 2 C_{J}$ takes the form of Eq. (1) with anharmonicity $K \approx E_{C} / \hbar$ and resonance frequency $\tilde{\omega}_{0} \approx 1 / \sqrt{L_{J} C_{J}}$. Also for this type of resonators the coupling rate $\kappa$ to the transmission line can be designed independently of $E_{J}$ and $E_{C}$ by designing an appropriate capacitive network. Similarly as for the transmission line JPA, the description in terms of the effective Hamiltonian Eq. (1) is based on the assumption that for relevant resonator fields the phase drop across the Josephson junctions is small (compare Eq. (30)). In the following section we study the validity of this approximation when the resonator is driven close to the bifurcation point where we expect parametric amplification to occur and analyze its implications for realizing a parametric amplifier with large bandwidth and dynamic range.

\section{Bandwidth and dynamic range constraints}

\subsection{Validity of the quartic approximation}

In Section 3.3 we have shown that the dynamic range of the JPA scales with $\kappa /|K|$. Also the bandwidth becomes larger with increasing $\kappa$, which indicates that a large $\kappa$ is desirable for JPAs. However, there are limitations on the maximal possible value for $\kappa$ as discussed in the following.

For deriving the Hamiltonian in Eq. (1), or more generally Eq. (36), we have expanded the SQUID cosine potential to quartic order in the dimensionless flux variable $\Phi_{n} / \varphi_{0}$, where $\Phi_{n} \equiv \Phi(x=d)$ is the phase drop across the SQUID. To guarantee that this approximation holds when we operate the device in the parametric amplification regime, we have to make sure that $\Phi_{n} / \varphi_{0}$ is small even when it is driven close to the bifurcation point. This is equivalent to keeping the current flowing through the SQUID small compared to the critical current.

To characterize the validity of the low order expansion of the cosine potential, we define the maximal coherent field inside the resonator $\alpha_{\max }$ as the one for which $\Phi_{n}=\varphi_{0}$. This is the coherent amplitude, at which the current flowing through the SQUID equals its critical current. According to Eq. (35) and Eq. (24) a coherent field $\alpha$ in mode $j$ leads to a maximal amplitude of $\Phi_{n}=\phi_{\mathrm{zp}, j} 2 \alpha \cos \left(k_{j} d\right)$ across the tunnel junctions, based on which we define the critical amplitude as

$$
\alpha_{\mathrm{max}, j}=\frac{\varphi_{0}}{\phi_{\mathrm{zp}, j}} \frac{1}{2 \cos \left(k_{j} d\right)}=\frac{\varphi_{0}}{\phi_{\mathrm{zp} \mathrm{f}, j}} \frac{l d}{2 L_{J} k_{j} d \sin \left(k_{j} d\right)} .
$$

The low order expansion of the cosine potential is only valid if the field inside the resonator $\alpha$ is much smaller than this maximal amplitude $\alpha<\alpha_{\max , j}$. In Section 3.1 we have found that the photon number in a resonator mode at the bifurcation point is $N_{\text {crit }}=(\kappa+\gamma) / \sqrt{3} K$. The ratio between $N_{\text {crit }}$ and the maximal coherent photon number $N_{\max , j} \equiv\left|\alpha_{\text {max }, j}\right|^{2}$ is therefore given by

$$
\frac{N_{\text {crit }}}{N_{\text {max }, j}}=\frac{8 \kappa}{\sqrt{3}} \frac{\hbar}{E_{\mathrm{J}} \cos ^{2}\left(k_{j} d\right)}\left(\frac{\varphi_{0}}{\phi_{\mathrm{zpf}}}\right)^{2} .
$$




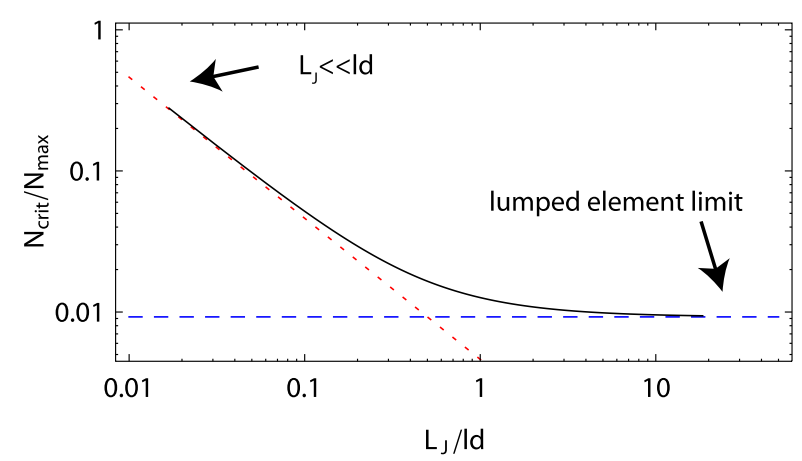

Figure 7 Validity of the quartic approximation. Ratio $N_{\text {crit }} / N_{\max }$ according to Eq. (40) (black), in the lumped element limit (dashed blue) and in the limit of small participation ratio $L_{j} \ll / d$ (dotted red) for the parameters $\left\{\tilde{\omega}_{0} / 2 \pi, Q\right\}=\{7 \mathrm{GHz}, 1,000\}$.

In order to minimize the effect of higher order nonlinearities we want to keep this ratio small. It has the following two interesting limits

$$
\frac{N_{\text {crit }}}{N_{\max , j}}= \begin{cases}\frac{8}{\sqrt{3}} Q^{-1} \frac{l d}{L_{J}}, & \text { for } L_{J} \ll l d \\ \frac{16}{\sqrt{3}} Q^{-1}, & \text { for lumped element JPA. }\end{cases}
$$

This is an important result, which sets clear constraints on both the maximally achievable bandwidth and the dynamic range of the JPA. If we want $N_{\text {crit }} / N_{\text {max }, j}$ to be small, $\kappa$ needs to be sufficiently small as well. One may also want to increase the dynamic range by reducing the Kerr nonlinearity $|K|$. While this can in principle be achieved by choosing a small ratio $L_{J} / l d$ between Josephson and geometric inductance, care has to be taken when using this approach because additional geometric inductance leads to a larger ratio $N_{\text {crit }} / N_{\max }$ as illustrated in Figure 7.

Interestingly, we find that in the lumped element case the Josephson inductance $L_{J}$, and with it the Kerr nonlinearity $K$, can in principle be made smaller without affecting $N_{\text {crit }} / N_{\text {max }}$. However, in practice a small Josephson inductance has to be compensated by a large lumped element capacitor to retain the desired resonance frequency, which is challenging to realize without introducing additional parasitic geometric inductances. It therefore seems difficult to build a parametric amplifier with large bandwidth and high dynamic range at the same time using a single SQUID only. In the following we show how one can keep $N_{\text {crit }} / N_{\max }$ constant while decreasing the nonlinearity and thus increasing the dynamic range of the amplifier, by replacing the single SQUID with a serial array of $M$ SQUIDs of $M$-times larger Josephson energy per SQUID (Figure 8).

\subsection{Josephson junction arrays}

For simplicity we assume that all SQUIDs in the array have the same effective Josephson energy $M E_{J}$. Since the spatial extent of the junction is still small compared to typical resonance wavelengths, we can treat the array as a lumped element. To derive the nonlinearity of the oscillator for this situation we investigate how the different terms in the Lagrangian scale with $M$.

Assuming that the phase drop from the flux node at the end of the transmission line resonator to the ground is homogeneously distributed over the array, we have the same 


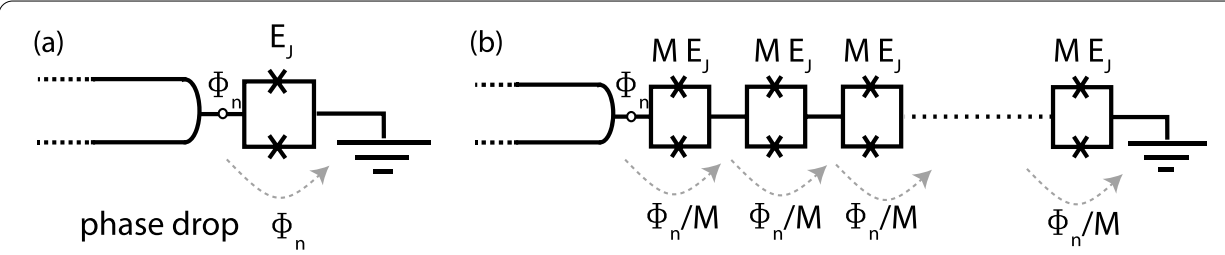

Figure 8 Schematic of the SQUID array. (a) The phase drop across a single SQUID junction is proportional to the node flux $\Phi_{n}$ (indicated by the circle) at the end of the transmission line. (b) If we replace the single junction by an serial array of $M$ junctions with $M$ times larger Josephson energy, the phase drop across each junction is by a factor of $M$ smaller while the total effective Josephson inductance stays the same.

phase drop $\Phi_{n} / M$ across each SQUID, see Figure 8. As a result the quadratic term in the Lagrangian scales as

$$
\frac{E_{\mathrm{J}}}{2} \Phi_{n}^{2} \stackrel{1 \rightarrow M}{\longrightarrow} \sum_{i=1}^{M} \frac{M E_{\mathrm{J}}}{2}\left(\frac{\Phi_{n}}{M}\right)^{2}=\frac{E_{\mathrm{J}}}{2} \Phi_{n}^{2}
$$

and thus remains constant. This agrees with our expectation, since the total linear Josephson inductance has not been changed. However, the quartic term scales like

$$
\frac{E_{\mathrm{J}}}{24} \Phi_{n}^{4} \stackrel{1 \rightarrow M}{\longrightarrow} \sum_{i=1}^{M} \frac{M E_{\mathrm{J}}}{24}\left(\frac{\Phi_{n}}{M}\right)^{4}=\frac{1}{M^{2}} \frac{E_{\mathrm{J}}}{24} \Phi_{n}^{4},
$$

which leads to a quadratic decrease in the effective Kerr nonlinearity $K \rightarrow K / M^{2}$ and thus a quadratic increase in $N_{\text {crit }} \propto M^{2}$. Furthermore, the maximal photon number also scales as $N_{\max } \propto M^{2}$ since the critical current of each junction is larger by a factor of $M$. In other words, the ratio $N_{\text {crit }} / N_{\max }$ only depends on the total Josephson inductance whereas the bifurcation power increases quadratically in $M$. We thus conclude that the dynamic range of a JPA can be increased without affecting the amplifier bandwidth, by using an array of SQUIDs instead of a single SQUID. This conclusion is valid for both the transmission line JPA and the lumped element JPA.

In practice, the Josephson energies in the array are not all equal due to inhomogeneous coupling to the external magnetic flux and scatter in the critical current of Josephson junctions due to unavoidable variations in fabrication. A quantitative analysis of the influence of such variations of Josephson energies on the parametric amplifier characteristics could be an interesting task for future studies. This would help to quantify limitations in the accessible tuning range of the parametric amplifier and a realistic understanding of the breakdown of the low order expansion of the cosine potential. For such an approach the methods used in Ref. [63] could turn out to be useful.

\section{Conclusion}

In summary, we have presented a detailed analysis of Josephson junction based parametric amplifiers, including a discussion of bandwidth, noise and dynamic range. By establishing relations between basic JPA properties and designable circuit parameters we have been able to derive two simple design strategies to achieve optimized JPA performance. On the one hand the contribution of the Josephson inductance to the total effective inductance of the resonant circuit has to be chosen sufficiently large. On the other hand the use of 
SQUID arrays instead of single SQUIDs provides the possibility to enhance the strength of the pump field close at the bifuraction point and with it the dynamic range of the JPA.

\section{Competing interests}

The authors declare they have no competing interests.

\section{Authors' contributions}

All authors contributed equally to the work in this article.

\section{Acknowledgements}

The authors would like to acknowledge helpful discussions with Alexandre Blais, Vitaly Shumeiko and Sebastian Schmidt. This work was supported by the European Research Council (ERC) through a Starting Grant and by ETHZ.

Received: 30 May 2013 Accepted: 25 September 2013 Published: 29 January 2014

\section{References}

1. Yurke B, Buks E: J Lightwave Technol 2006, 24:5054.

2. Castellanos-Beltran MA, Lehnert KW: Appl Phys Lett 2007, 91:083509

3. Tholén E, Ergül A, Doherty E, Weber F, Grégis F, Haviland D: Appl Phys Lett 2007, 90:253509.

4. Kinion D, Clarke J: Appl Phys Lett 2008, 92:172503.

5. Yamamoto T, Inomata K, Watanabe M, Matsuba K, Miyazaki T, Oliver WD, Nakamura Y, Tsai JS: Appl Phys Lett 2008, 93:042510

6. Castellanos-Beltran MA, Irwin KD, Hilton GC, Vale LR, Lehnert KW: Nat Phys 2008, 4:929.

7. Palacios-Laloy A, Nguyen F, Mallet F, Bertet P, Vion D, Esteve D: J Low Temp Phys 2008, 151:1034.

8. Kamal A, Marblestone A, Devoret M: Phys Rev B 2009, 79:184301.

9. Bergeal N, Schackert F, Metcalfe M, Vijay R, Manucharyan VE, Frunzio L, Prober DE, Schoelkopf RJ, Girvin SM, Devoret $\mathrm{MH}$ : Nature 2010, 465:64.

10. Hatridge M, Vijay R, Slichter DH, Clarke J, Siddiqi I: Phys Rev B 2011, 83:134501.

11. Vijay R, Slichter DH, Siddiqi I: Phys Rev Lett 2011, 106:110502.

12. Gao J, Vale LR, Mates JAB, Schmidt DR, Hilton GC, Irwin KD, Mallet F, Castellanos-Beltran MA, Lehnert KW, Zmuidzinas J, Leduc HG: Appl Phys Lett 2011, 98:232508.

13. Eichler C, Bozyigit D, Lang C, Baur M, Steffen L, Fink JM, Filipp S, Wallraff A: Phys Rev Lett 2011, 107:113601.

14. Abdo B, Kamal A, Devoret M: Phys Rev B 2013, 87:014508.

15. Kamal A, Clarke J, Devoret MH: Phys Rev B 2012, 86:144510

16. Louisell WH, Yariv A, Siegman AE: Phys Rev 1961, 124:1646.

17. Gordon JP, Louisell WH, Walker LR: Phys Rev 1963, 129:481.

18. Mollow BR, Glauber RJ: Phys Rev 1967, 160:1076.

19. Clerk AA, Devoret MH, Girvin SM, Marquardt F, Schoelkopf RJ: Rev Mod Phys 2010, 82:1155

20. Mallet F, Castellanos-Beltran MA, Ku HS, Glancy S, Knill E, Irwin KD, Hilton GC, Vale LR, Lehnert KW: Phys Rev Lett 2011 106:220502.

21. Flurin E, Roch N, Mallet F, Devoret MH, Huard B: Phys Rev Lett 2012, 109:183901.

22. Menzel EP, Di Candia R, Deppe F, Eder P, Zhong L, Ihmig M, Haeberlein M, Baust A, Hoffmann E, Ballester D, Inomata K, Yamamoto T, Nakamura Y, Solano E, Marx A, Gross R: Phys Rev Lett 2012, 109:250502.

23. Vijay R, Macklin C, Slichter DH, Weber SJ, Murch KW, Naik R, Korotkov AN, Siddiqi I: Nature 2012, $490: 77$.

24. Johnson JE, Macklin C, Slichter DH, Vijay R, Weingarten EB, Clarke J, Siddiqi I: Phys Rev Lett 2012, 109:050506.

25. Ristè D, van Leeuwen JG, Ku H-S, Lehnert KW, DiCarlo L: Phys Rev Lett 2012, 109:050507.

26. Campagne-Ibarcq P, Flurin E, Roch N, Darson D, Morfin P, Mirrahimi M, Devoret MH, Mallet F, Huard B: Phys Rev X 2013, 3:021008.

27. Teufel JD, Donner T, Li D, Harlow JW, Allman MS, Cicak K, Sirois AJ, Whittaker JD, Lehnert KW, Simmonds RW: Nature 2011, 475:359.

28. Eichler C, Bozyigit D, Wallraff A: Phys Rev A 2012, 86:032106.

29. Eichler C, Lang C, Fink JM, Govenius J, Filipp S, Wallraff A: Phys Rev Lett 2012, 109:240501.

30. Steffen L, Salathe Y, Oppliger M, Kurpiers P, Baur M, Lang C, Eichler C, Puebla-Hellmann G, Fedorov A, Wallraff A: Nature 2013, 500:319.

31. Louisell WH: Coupled Mode and Parametric Electronics. New York: John Wiley; 1960

32. Slusher RE, Hollberg LW, Yurke B, Mertz JC, Valley JF: Phys Rev Lett 1985, 55:2409.

33. Burnham DC, Weinberg DL: Phys Rev Lett 1970, 25:84.

34. Bergeal N, Vijay R, Manucharyan VE, Siddiqi I, Schoelkopf RJ, Girvin SM, Devoret MH: Nat Phys 2010, 6:296.

35. Bergeal N, Schackert F, Frunzio L, Devoret MH: Phys Rev Lett 2012, 108:123902.

36. Roch N, Flurin E, Nguyen F, Morfin P, Campagne-lbarcq P, Devoret MH, Huard B: Phys Rev Lett 2012, 108:147701.

37. Ho Eom B, Day PK, LeDuc HG, Zmuidzinas J: Nat Phys 2012, 8:623.

38. Yaakobi O, Friedland L, Macklin C, Siddiqi I: Phys Rev B 2013, 87:144301.

39. Wilson CM, Johansson G, Pourkabirian A, Simoen M, Johansson JR, Duty T, Nori F, Delsing P: Nature 2011, $479: 376$.

40. Wustmann W, Shumeiko V: Phys Rev B 2013, 87:184501.

41. Dykman M, Krivoglaz M: Phys A, Stat Mech App/ 1980, 104:480.

42. Marthaler M, Dykman Ml: Phys Rev A 2006, 73:042108.

43. Siddiqi I, Vijay R, Pierre F, Wilson CM, Metcalfe M, Rigetti C, Frunzio L, Devoret MH: Phys Rev Lett 2004, 93:207002.

44. Vijay R, Devoret MH, Siddiqi I: Rev Sci Instrum 2009, 80:111101.

45. Mallet F, Ong FR, Palacios-Laloy A, Nguyen F, Bertet P, Vion D, Esteve D: Nat Phys 2009, 5:791.

46. Gardiner CW, Collett MJ: Phys Rev A 1985, 31:3761. 
47. Walls DF, Milburn GJ: Quantum Optics. Berlin: Springer; 1994.

48. Göppl M, Fragner A, Baur M, Bianchetti R, Filipp S, Fink JM, Leek PJ, Puebla G, Steffen L, Wallraff A: J Appl Phys 2008, 104:113904.

49. Braunstein SL, van Loock P: Rev Mod Phys 2005, 77:513.

50. Caves CM: Phys Rev D 1982, 26:1817.

51. Wallquist M, Shumeiko VS, Wendin G: Phys Rev B 2006, 74:224506.

52. Bourassa J, Beaudoin F, Gambetta JM, Blais A: Phys Rev A 2012, 86:013814.

53. Girvin SM: Lectures delivered at Ecole d'Eté Les Houches. To be published by Oxford University Press, 2011.

54. Imoto N, Haus HA, Yamamoto Y: Phys Rev A 1985, 32:2287.

55. Sanders BC, Milburn GJ: Phys Rev A 1989, 39:694.

56. Santamore DH, Goan H-S, Milburn GJ, Roukes ML: Phys Rev A 2004, 70:052105.

57. Buks E, Yurke B: Phys Rev A 2006, 73:023815.

58. Helmer F, Mariantoni M, Solano E, Marquardt F: Phys Rev A 2009, 79:052115.

59. Johnson BR, Reed MD, Houck AA, Schuster DI, Bishop LS, Ginossar E, Gambetta JM, DiCarlo L, Frunzio L, Girvin SM, Schoelkopf RJ: Nat Phys 2010, 6:663.

60. Suchoi O, Abdo B, Segev E, Shtempluck O, Blencowe MP, Buks E: Phys Rev B 2010, 81:174525.

61. Kirchmair G, Vlastakis B, Leghtas Z, Nigg SE, Paik H, Ginossar E, Mirrahimi M, Frunzio L, Girvin SM, Schoelkopf RJ: Nature 2013, 495:205

62. Koch J, Yu TM, Gambetta J, Houck AA, Schuster DI, Majer J, Blais A, Devoret MH, Girvin SM, Schoelkopf RJ: Phys Rev A 2007, 76:042319.

63. Ferguson DG, Houck AA, Koch J: Phys Rev X 2013, 3:011003.

doi:10.1140/epjqt2

Cite this article as: Eichler and Wallraff: Controlling the dynamic range of a Josephson parametric amplifier. EPJ

Quantum Technology 2014 1:2

\section{Submit your manuscript to a SpringerOpen ${ }^{\circ}$ journal and benefit from:}

- Convenient online submission

Rigorous peer review

- Immediate publication on acceptance

- Open access: articles freely available online

- High visibility within the field

- Retaining the copyright to your article 